\title{
Modeling risk of coronary obstruction during transcatheter aortic valve replacement
}

Megan Heitkemper, MS, ${ }^{\mathrm{a}}$ Hoda Hatoum, $\mathrm{PhD},{ }^{\mathrm{a}}$ Amirsepehr Azimian, MS, ${ }^{\mathrm{a}}$ Breandan Yeats, BS, ${ }^{\mathrm{a}}$ Jennifer Dollery, BSn, ${ }^{\mathrm{b}}$ Bryan Whitson, MD, PhD, ${ }^{\mathrm{b}}$ Greg Rushing, MD, ${ }^{\mathrm{b}}$ Juan Crestanello, MD, ${ }^{\mathrm{a}, \mathrm{b}}$ Scott M. Lilly, MD, PhD, ${ }^{\mathrm{c}}$ and Lakshmi Prasad Dasi, $\mathrm{PhD}^{\mathrm{a}, \mathrm{b}}$

\section{ABSTRACT}

Objective: In this study we aimed to evaluate risk of coronary obstruction during transcatheter aortic valve replacement and develop improved criteria based on computational modeling.

Methods: Patient specific 3-dimensional models were constructed and validated for 28 patients out of 600 patients who were flagged as high risk for coronary obstruction (defined as meeting coronary ostium height $<14 \mathrm{~mm}$ and/or sinus of Valsalva diameter [SOVd] $<30 \mathrm{~mm}$ ). The models consisted finite element analysis to predict the post- transcatheter aortic valve replacement native cusp apposition relative to the coronary ostium and were validated in vitro. The distance from cusp to coronary ostium (DLC) was derived from the 3-dimensional models and indexed with the coronary artery diameter to yield a fractional obstruction measure (DLC/d).

Results: Twenty-two out of 28 high-risk patients successfully underwent transcatheter aortic valve replacement without coronary obstruction and 6 did not. DLC/d between the 2 groups was significantly different $(P<.00078)$, whereas neither coronary ostium height nor SOVd were significantly different $(P>.32)$. A cutoff of DLC/d $<0.7$ was predictive with $100 \%$ sensitivity and $95.7 \%$ specificity. The optimal sensitivity and specificity of coronary ostium height and SOVd in this high-risk group was only $60 \%$ and $40 \%$, respectively, for cutoff coronary ostium height of $10 \mathrm{~mm}$ and SOVd of $30.5 \mathrm{~mm}$.

Conclusions: Three-dimensional modeling has the potential to enable more patients to be safely treated with transcatheter aortic valve replacement who have a low-lying coronary ostium or small SOVd. DLC/d is more predictive of obstruction than coronary ostium height and SOVd. (J Thorac Cardiovasc Surg 2020;159:829-38)

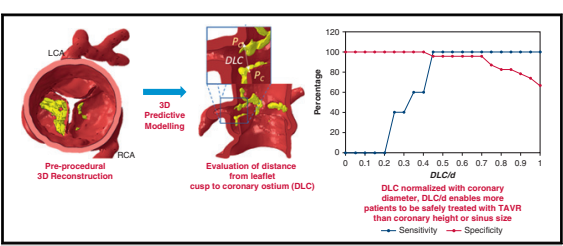

3D model-generated prediction of coronary obstruction using the new DLC parameter.

Central Message

A significant number of patients with low-lying coronary height are not at risk for coronary obstruction. 3D modeling can help bring the benefit of TAVR to these patients.

\section{Perspective}

Coronary ostium height and sinus of Valsalva size are not predictive of successful TAVR in patients with low-lying coronary height. 3D modeling-generated parameters that use the predicted distance between the cusp and coronary ostium are predictive of whether or not TAVR will be successful.

See Commentaries on pages 839 and 842.
Transcatheter aortic valve replacement (TAVR) represents a major advance in the field of cardiac surgery and interventional cardiology for the treatment of patients with severe aortic stenosis, for whom conventional heart surgery has been deemed high risk. ${ }^{1-4}$ Despite the overall effectiveness of TAVR, complications can limit the realization of mortality and quality of life benefits. ${ }^{4-7}$ Among these is coronary obstruction, which can occur upon transcatheter valve deployment, and most often

\footnotetext{
From the Departments of ${ }^{a}$ Biomedical Engineering and ${ }^{b}$ Surgery, and ${ }^{c}$ Division of Cardiology, The Ohio State University, Columbus, Ohio.

Received for publication Feb 12, 2018; revisions received April 6, 2019; accepted for publication April 16, 2019; available ahead of print June 21, 2019.

Address for reprints: Lakshmi Prasad Dasi, PhD, Departments of Biomedical Engineering and Surgery, The Ohio State University, Room 405A DHLRI, 473 W 12th Ave, Columbus, OH 43210 (E-mail: lakshmi.dasi@osumc.edu). $0022-5223 / \$ 36.00$

Copyright (c) 2019 by The American Association for Thoracic Surgery https://doi.org/10.1016/j.jtcvs.2019.04.091
}

affects the left coronary artery (LCA). ${ }^{8-15}$ Coronary obstruction, as defined by the 2011 American College of Cardiology Foundation/American Heart Association Task Force on Practice Guidelines and the Society for Cardiovascular Angiography and Interventions guidelines for percutaneous coronary intervention, is considered as a $>50 \%$ obstruction of the left main coronary artery, $>70 \%$ in any other coronary artery, or both. ${ }^{16,17}$ Although this complication is rare (reported in up to $1 \%$ of all TAVR procedures) the outcomes can often be catastrophic. Proper coronary flow has also been shown to enhance

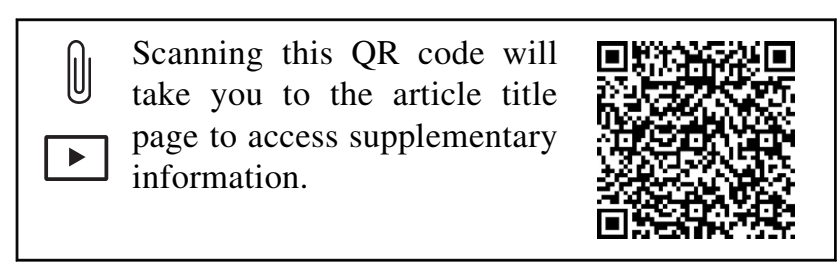




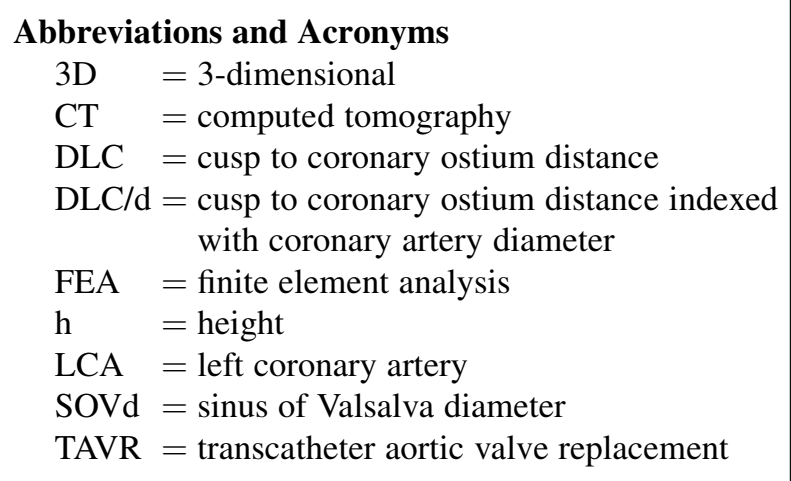

sinus flow and washout in the context of mitigating leaflet thrombosis after TAVR. ${ }^{18,19}$

Although a serious and potentially preventable complication, there is no consensus to which features reliably predispose risk of coronary obstruction during TAVR. Most of the guidelines developed so far have originated from clinical trials designed to exclude as many adverse outcomes as possible and were not based on simulations or mechanistic insights into the precise mechanics of coronary obstruction. In doing so, these guidelines have potential to exclude a large number of potential TAVR patients, often those who have no other treatment options available.

Despite the existing predictive models, $1 \%$ to $3 \%$ of TAVR patients still experience coronary obstruction. However, it has been shown that restrictively applying the current guidelines could have excluded $26 \%$ to $33 \%$ of patients who successfully received TAVR with no reported instance of coronary obstruction. ${ }^{20}$ In an in-vitro study that examined how sensitive coronary obstruction risk is to valve types, it was shown that a different selection of valve types and/or sizes could have mitigated the risk. $^{21}$ This clearly demonstrates the importance of patient-specific modeling and the critical need for individualization of valve replacement therapy.

The objective of this study is to better understand the physical mechanism of coronary obstruction beyond the conventional parameters of coronary ostium height and sinus of Valsalva diameter (SOVd) alone and introduce a new, more accurate mechanistic index that can predict which high risk patients (ie, patients with coronary ostium height $<14 \mathrm{~mm}$ and/or SOVd $<30 \mathrm{~mm}$ ) are not actually at risk and are indeed candidates for TAVR preoperatively, allowing most patients to safely undergo TAVR without coronary obstruction.

\section{METHODS}

To better understand the mechanism of coronary obstruction and develop a mechanistic index that can predict which high-risk patients (ie, patients with coronary ostium height $<14 \mathrm{~mm}$ and/or SOVd $<30 \mathrm{~mm}$ ) are not actually at risk and are indeed candidates for TAVR preoperatively, a 3-dimensional (3D) computational model that uses pre-TAVR computed tomography $(\mathrm{CT})$ angiogram imaging is presented and compared with the conventional guidelines. The 3D model employs computer-aided methodologies that predict the closest distance between native aortic valve cusp and the corresponding coronary artery ostium following TAV deployment. In vitro validation of this novel computational model was performed using 3D printed flexible patient-specific aortic root geometries. Informed consent was obtained from all patients and the study complied with the Institutional Review Board of The Ohio State University.

\section{Study Population}

The study population included all moderate to high-risk patients, defined by LCA height $(\mathrm{LCAh})<14 \mathrm{~mm}$ and/or SOVd $<30 \mathrm{~mm}$, flagged from 600 aortic stenosis patients considered for TAVR at The Ohio State University Wexner Medical Center between January 2014 and September 2018. This filtering resulted in 28 patients (patients A-AB in Table 1) being flagged as moderate to high risk for LCA obstruction during TAVR and included $78.5 \%$ women aged $80 \pm 9$ years with symptomatic severe aortic stenosis. The individual LCAh and SOVd are shown in Figure 1 for the study population with quadrants representing LCAh $<12 \mathrm{~mm}$ and SOVd $<30 \mathrm{~mm}$ based on the analysis provided by Ribeiro and colleagues ${ }^{20}$ (Figure 1 is discussed later).

With respect to the outcomes for these 28 patients, 23 received TAVR successfully, whereas 5 patients did not receive a successful TAVR. These 5 include 1 man who experienced coronary obstruction (Video 1), 2 women who underwent surgical aortic valve replacement with visual confirmation of coronary obstruction by the operating surgeon, and 1 man and 1 woman (patients $\mathrm{H}$ and $\mathrm{V}$ ) who each had extremely low-lying coronary ostium ( $9 \mathrm{~mm}$ and $8 \mathrm{~mm}$, respectively) and were deemed surgically inoperable due to age and received medical management.

\section{D Computational Model}

A 3D computational model was developed to assess risk of coronary obstruction during TAVR. This model is only for patients who already satisfy a conservative risk stratification (ie, coronary ostium height $<14 \mathrm{~mm}$ and/or SOVd $<30 \mathrm{~mm}$ ) and as is shown later, there would not be any benefit to performing $3 \mathrm{D}$ computational modeling for lower-risk patients. The model works by simulating the implantation of an idealized and cylindrical TAV prosthesis into a patient's preprocedural aortic root anatomy (including the calcified native cusps). The risk for coronary obstruction is then assessed through quantifying the closest distance of the cusp and the corresponding coronary ostium. This distance is indexed to the coronary artery diameter to obtain a representative measure of the fractional obstruction of the native cusp eclipsing the ostium.

The preprocedure patient-specific aortic root, calcium nodules, and cusps were segmented for each of the 28 patients from pre-TAVR CT images using Mimics Research 18.0 (Materialise, Leuven, Belgium). The segmented aortic wall, cusps, and calcium nodules were then discretized in 3-Matic Research 13.0 (Materialise) using explicit 4-node linear tetrahedron elements (Figure E1). An idealized TAV stent (represented as an expandable cylinder) was discretized using hexahedral elements.

An example of the segmented aortic root and cusp with calcification anatomy previous to TAV implantation is depicted in Figure 2, $A$ through $C$. Finite element analysis (FEA) was performed on each patient-specific 3D anatomic model using Abaqus/Explicit 6.9 software (Simulia, Providence, RI) to simulate the opening of a TAV device stent that pushes the native cusps open toward the coronary ostium. For each patient's anatomy, the simulation expanded the TAV device stent to the diameter of the valve size that would be appropriate for that patient's anatomy, as determined by the structural heart team at The Ohio State University Wexner Medical Center. The simulated TAV expanded diameters and the valve sizes received are given in Table 1. Material properties of the pressurized aortic 
TABLE 1. List of coronary obstruction predictive parameters, including currently used parameters namely coronary ostium height, sinus of Valsalva diameter, and newly proposed predictive parameters based on the 3-dimensional computational modeling for each patient

\begin{tabular}{|c|c|c|c|c|c|c|c|c|c|c|}
\hline Patient & Sex & Age & $\begin{array}{c}\text { LCA } \\
\text { height } \\
(\mathbf{m m})\end{array}$ & $\begin{array}{c}\text { Sinus of } \\
\text { valsava } \\
\text { diameter }(\mathbf{m m})\end{array}$ & $\begin{array}{c}\text { LCA } \\
\text { diameter } \\
(\mathbf{m m})\end{array}$ & $\begin{array}{c}\text { Valve } \\
\text { diameter } \\
(\mathbf{m m})\end{array}$ & $\begin{array}{c}\text { Simulated } \\
\text { TAV expansion } \\
\text { diameter }(\mathbf{m m})\end{array}$ & DLC & DLC/d & $\begin{array}{c}\text { TAVR } \\
\text { Successful? }\end{array}$ \\
\hline A & Male & 86 & 13 & 32 & 3.6 & 25 & 25 & 3.5 & 1.0 & Yes \\
\hline B & Female & 70 & 12 & 27 & 5.4 & 23 & 23 & 7.4 & 1.4 & Yes \\
\hline $\mathrm{C}$ & Male & 88 & 7 & 36 & 5.3 & 26 & 26 & 2.3 & 0.4 & Yes \\
\hline D & Female & 89 & 9 & 30 & 5.5 & 23 & 23 & 3.9 & 0.7 & Yes \\
\hline $\mathrm{E}$ & Male & 93 & 13 & 30 & 2.8 & 23 & 23 & 3.0 & 1.1 & Yes \\
\hline $\mathrm{F}$ & Female & 79 & 10 & 30 & 3.3 & 23 & 23 & 3.3 & 1.0 & Yes \\
\hline G & Female & 81 & 12 & 29 & 5.6 & 26 & 26 & 5.7 & 1.0 & Yes \\
\hline $\mathrm{H}$ & Male & 94 & 9 & 32 & 4.6 & NA & 26 & 0.9 & 0.2 & No \\
\hline I & Female & 81 & 9 & 30 & 4.0 & 29 & 29 & 2.8 & 0.7 & Yes \\
\hline $\mathrm{J}$ & Female & 75 & 9 & 28 & 5.7 & NA & 23 & 1.2 & 0.2 & No \\
\hline K & Male & 80 & 12 & 37 & 5.4 & 29 & 29 & 1.8 & 0.3 & No \\
\hline $\mathrm{L}$ & Female & 68 & 8 & 26 & 4.6 & 23 & 23 & 3.6 & 0.8 & Yes \\
\hline M & Female & 88 & 13 & 30 & 5.0 & 29 & 29 & 7.0 & 1.4 & Yes \\
\hline $\mathrm{N}$ & Female & 91 & 19 & 28 & 3.6 & 29 & 29 & 9.1 & 2.5 & Yes \\
\hline $\mathrm{O}$ & Female & 81 & 9 & 31 & 3.1 & 29 & 29 & 3.7 & 1.2 & Yes \\
\hline $\mathrm{P}$ & Female & 77 & 9 & 31 & 3.2 & 23 & 23 & 5.0 & 1.6 & Yes \\
\hline Q & Female & 87 & 19 & 27 & 3.2 & 20 & 20 & 12.6 & 3.9 & Yes \\
\hline $\mathrm{R}$ & Female & 74 & 12 & 33 & 4.6 & 23 & 23 & 4.2 & 0.9 & Yes \\
\hline$S$ & Female & 84 & 9 & 31 & 4.2 & 26 & 26 & 6.4 & 1.5 & Yes \\
\hline $\mathrm{T}$ & Female & 62 & 11 & 29 & 4.4 & NA & 23 & 1.9 & 0.4 & No \\
\hline $\mathrm{U}$ & Female & 77 & 9 & 26 & 4.2 & 26 & 26 & 4.0 & 1.0 & Yes \\
\hline V & Female & 91 & 8 & 31 & 4.6 & NA & 23 & 1.8 & 0.4 & No \\
\hline W & Female & 82 & 11 & 27 & 3.2 & 23 & 23 & 3.6 & 1.1 & Yes \\
\hline$X$ & Female & 72 & 13 & 29 & 4.9 & 26 & 26 & 7.9 & 1.6 & Yes \\
\hline $\mathrm{Y}$ & Female & 76 & 12 & 33 & 3.4 & 23 & 23 & 5.0 & 1.5 & Yes \\
\hline Z & Male & 61 & 9 & 29 & 2.6 & 26 & 26 & 7.0 & 2.7 & Yes \\
\hline AA & Female & 83 & 10 & 30 & 2.8 & 23 & 23 & 3.9 & 1.4 & Yes \\
\hline $\mathrm{AB}$ & Female & 77 & 13 & 28 & 2.7 & 23 & 23 & 6.3 & 2.3 & Yes \\
\hline
\end{tabular}

$L C A$, Left coronary artery; $T A V$, transcatheter aortic valve; $D L C$, distance from cusp to coronary ostium; $D L C / d$, the fraction of distance between the aortic cusp and coronary ostium post-TAV deployment available for blood flow toward the coronary ostium; TAVR, transcatheter aortic valve replacement.

root were assigned using an isotropic neo-Hookean hyperelastic model based on the studies by Bosmons and colleagues, ${ }^{22}$ and Auricchio and colleagues. ${ }^{23}$ The strain energy function is described as

$$
W=\frac{\mu}{2}\left(I_{1}-3-0.5 \operatorname{Ln} J\right)+\frac{\lambda}{2}(\operatorname{Ln} J)^{2}
$$

Where $\mu$ and $\lambda$ are shear and bulk modulus, respectively, and are shown in Table E1 for each part. Calcium nodules were approximated to be linear elastic. The Young's modulus was based on the nonlinear elastic material properties introduced by Billiar and Sacks. ${ }^{24}$

Figure 2, $D$, shows a schematic of the postsimulation anatomy highlighting the closest distance between the left cusp and corresponding coronary ostium. The predicted gap (ie, the distance from the cusp to the coronary ostium [DLC]) would be seen in a long axis plane showing both the coronary ostium and the native cusp. Figure 2, $E$ and $F$, show different 3D perspectives of the postsimulation anatomy for the same distance (ie, DLC) from a cross-sectional and top view for the same patient. DLC was then normalized with respect to the corresponding coronary artery diameter to obtain a measure of the distance between the aortic cusp and coronary ostium post-TAV deployment available for blood flow toward the coronary ostium (DLC/d). A fractional value greater than unity indicates that the gap available for blood flow is greater than the coronary artery diameter. A fractional value approaching zero indicates total occlusion. Figure E2 visualizes the same for 4 patients.

\section{In Vitro Validation}

The computational model was validated in vitro as well as in vivo. Two flexible patient-specific 3D printed models of aortic root geometries, the first with 3D printed patient calcium nodules, and the second without calcium nodules, were used to evaluate the potential effects of rigid calcium nodules on cusps' deformation during TAVR. The 3D printed aortic root model was manufactured using a Connex 350 3D printer (Stratasys, Farmington Hills, Mich) from TangoPlus (Stratasys) material for the aorta and cusps and VeroWhite (Stratasys) material used for the calcium nodules (Figure 3). The flexible 3D printed model with the 


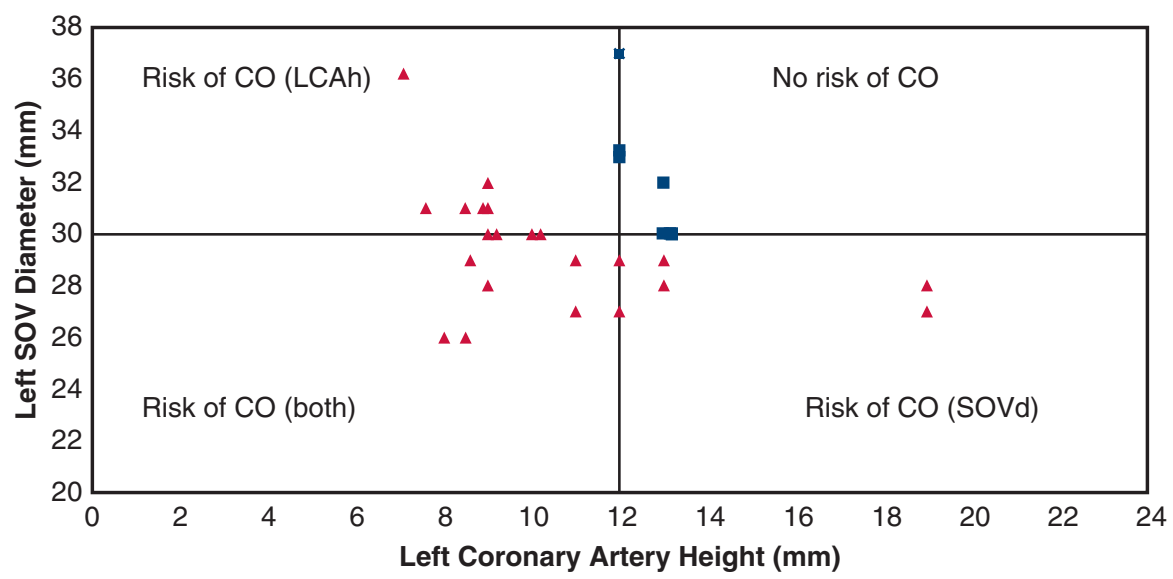

FIGURE 1. Study population characterized by conventional parameters (coronary height $[\mathrm{h}]<14 \mathrm{~mm}$ and sinus of Valsalva diameter [SOVd] $<30 \mathrm{~mm}$ ) used to predict left coronary artery obstruction before transcatheter aortic valve replacement (TAVR) with origin located at (12,30), representing a left coronary artery height $(L C A h)$ of $12 \mathrm{~mm}$ and a left a sinus of Valsalva diameter (SOVd) of $30 \mathrm{~mm}$. Blue squares represent the only patients who would have been approved for TAVR under these current guidelines. SOV, Sinus of Valsalva diameter; CO, coronary obstruction.

calcification nodules itself was also validated through comparison of patient hemodynamic parameters (ie, peak gradients and velocities) with experimental values obtained from left heart flow simulator studies. ${ }^{25,26}$ An idealized tool (Figure 3,F) was used to open the cusps to the appropriate stent size for the 3D printed models and was compared against the results of the FEA simulation of the same patient with and without inclusion of calcium nodules. As shown in Figure E3, there was excellent agreement between the computational prediction and experimental measurements of DLC.

Further validation of the 3D computational approach was achieved with the observation of coronary occlusion to occur in 2 patients $(\mathrm{J}$ and $\mathrm{T}$ in Table 1) as predicted by the model and confirmed by the operating surgeon during surgical aortic valve replacement.

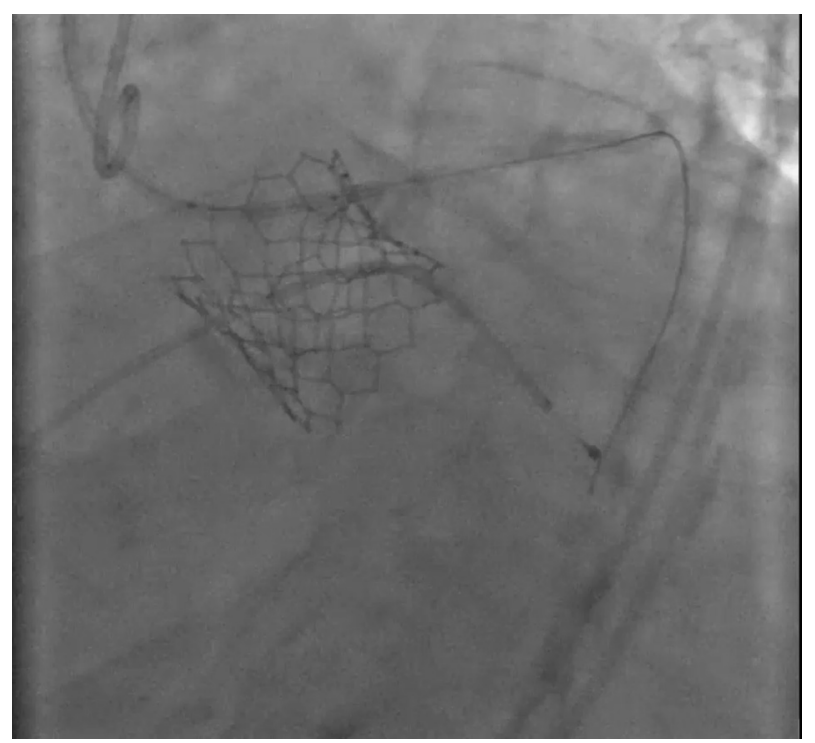

VIDEO 1. Coronary obstruction occurrence, patient K (Table 1). Video available at: https://www.jtcvs.org/article/S0022-5223(19)31030-X/fulltext.

\section{Statistical Analysis}

A Mann-Whitney nonparametric comparison of means was performed for each of the 3 parameters (DLC/d, coronary ostium height, and SOVd) to compare the mean parameter value between the 2 groups: the 23 who underwent TAVR successfully and the 5 patients who did not undero successful TAVR. A sensitivity and specificity analysis was performed for each test (DLC/d, coronary artery height, and SOVd) by identifying how many patients would identify as true positive, false positive, true negative, or false negative for coronary obstruction under a range of cutoff values. Sensitivity was calculated as

$$
\text { Sensitivity }=\frac{\text { True positives }}{\text { True positives }+ \text { False negatives }},
$$

Whereas specificity was calculated as

$$
\text { Specificity }=\frac{\text { True negatives }}{\text { True negatives }+ \text { False positives }},
$$

as described in Lalkhen and McCluckey. ${ }^{27}$

\section{RESULTS}

Here we present results that compare the ability of solely conventional parameters such as coronary ostium height and SOVd and the new parameter DLC/d to differentiate which high-risk patients are not actually at risk and are indeed candidates for TAVR. Routine anatomic measurements of coronary ostium height and SOVd along with measured values from the 3D computational model, DLC, and DLC/d for the high risk study population (28 patients) are presented in Table 1 .

\section{Current Guidelines}

Figure 1 shows the risk assessment for left coronary obstruction occurrence under the existing guidelines (ie, coronary ostium height $<12 \mathrm{~mm}$ and SOVd $<30 \mathrm{~mm}$ ) based on Ribeiro and colleagues. ${ }^{20}$ Obstruction of the right coronary artery was not evaluated due to lack of right coronary obstruction in our patient population. 


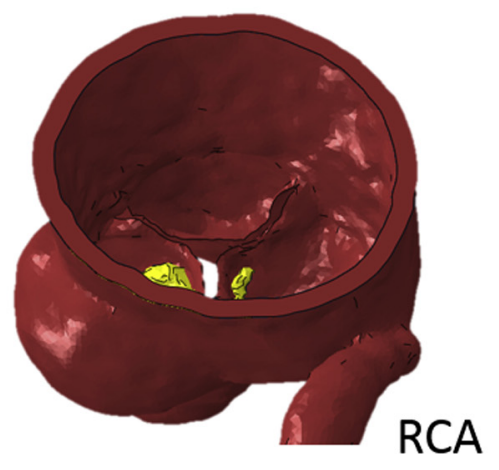

A

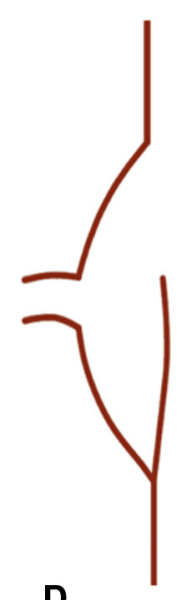

D

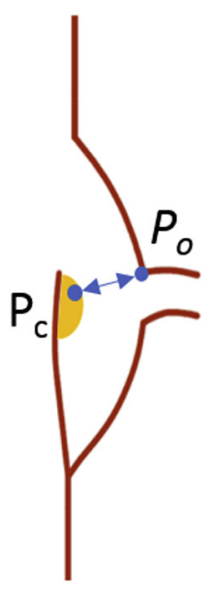

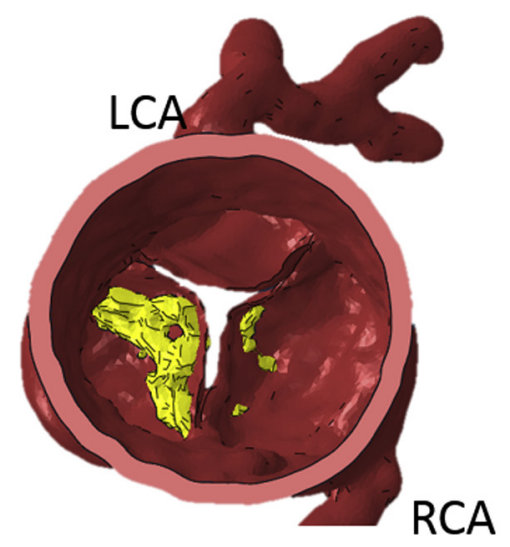

B

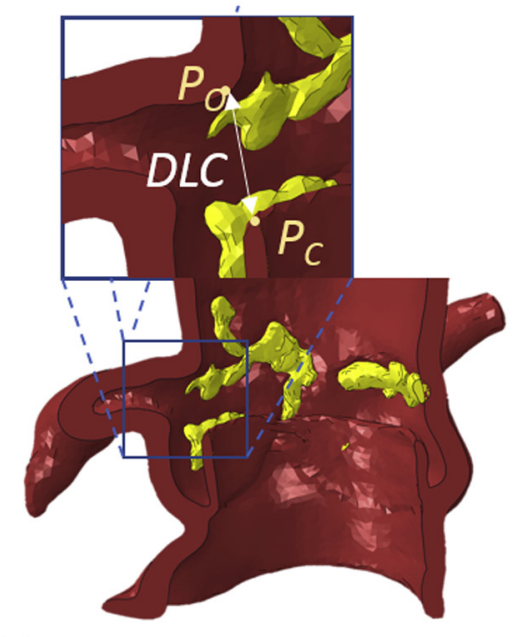

E

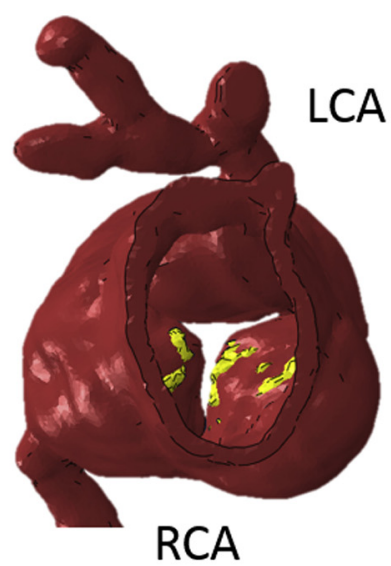

C

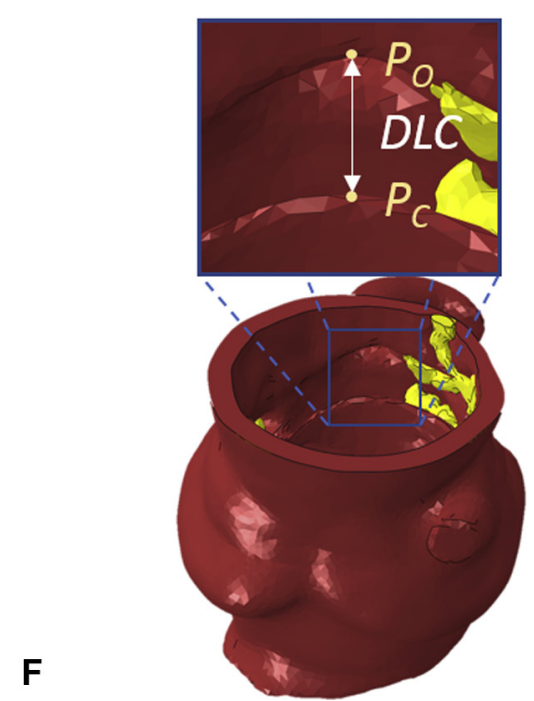

FIGURE 2. Example of patient specific 3-dimensional modeled aortic root with left coronary artery $(L C A)$, right coronary artery $(R C A)$, and yellow calcific nodules. A, Side view; B, aortic view; C, ventricular view. D, The measured distance $(D L C)$ from a point on cusp/or cusp calcium $(P C)$ to a point on the upper ostium of the coronary artery $(P o)$ following a transcatheter valve replacement from the idealized root schematic from the side view. E, Example finite element simulated post-transcatheter aortic valve replacement aortic root with DLC from a top view. F, Side view.

Accordingly, left coronary obstruction was expected to occur for 22 of the 28 patients, with left SOVd in the range of 26 to $36 \mathrm{~mm}$ and LCAh in the range of 7 to $19 \mathrm{~mm}$.

\section{D Predictive Model}

The distribution of DLC/d values among the patient population is shown in Figure 4. The range of values for DLC/d for patients was between 0.2 and 3.9. The horizontal line between 0.5 and 0.7 approximately separates patients who successfully received TAVR (above the line) from those who did not. The 1 blue data point that lies just below the horizontal line represents a patient who successfully received TAVR but only with coronary protection using a stent.

\section{Comparison With Current Guidelines}

The parameter values for those high-risk patients who successfully received TAVR without coronary obstruction are compared with those who did not receive TAVR successfully in Figure 5. The means for these 2 groups were compared using a Mann-Whitney nonparametric test, and the only significant difference between the 2 groups was found for the DLC/d parameter, with $P<.00078$. Neither LCAh nor SOVd was significantly different between the 2 groups $(P=.35238$ and $P=.32218$, respectively).

Figure 6 shows sensitivity and specificity curves generated for each of the 3 parameters to accurately predict whether TAVR within this high-risk patient population 

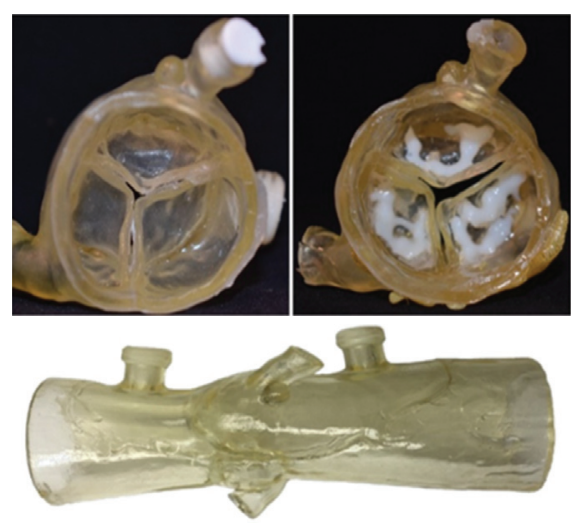

A

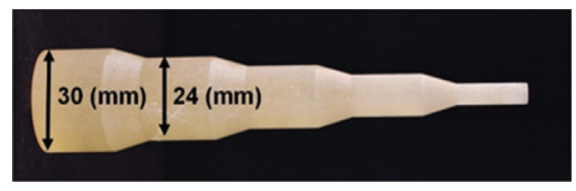

$\mathbf{F}$

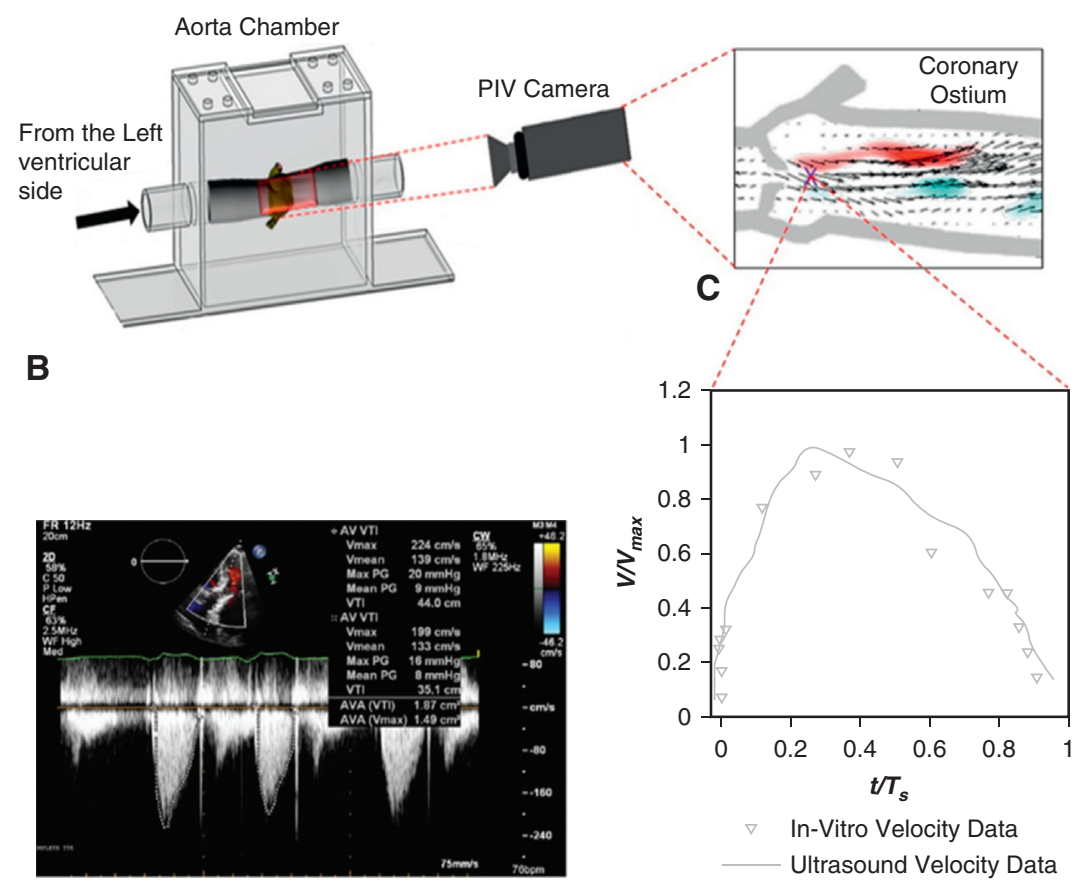

D

FIGURE 3. A, The 3-dimensional (3D) printed aortic root model was manufactured from TangoPlus (Stratasys, Farmington Hills, Mich) material and VeroWhite (Stratasys) material was used for calcium nodule, both printed using Connex 350 3D printer (Stratasys). B, Particle image velocimetry (PIV) experiments were performed to validate the 3D printed calcified aortic root model and compare with in vivo ultrasound Doppler jet velocity for the patient. Detailed methodology of the PIV experiments may be found in Hatoum and colleagues ${ }^{28}$ and Hatoum and colleagues. ${ }^{29}$ C, Comparison of the temporal velocity profile corresponding to a point located at the exit of the systolic jet of the valve. D and E, Doppler data. As can be seen, the result shows good agreement between the in vivo and in vitro data. The maximum velocity in the PIV data was found to be $2.10 \mathrm{~m} / \mathrm{second}$, which compares well with $2.24 \mathrm{~m} / \mathrm{second}$ obtained from the ultrasound. F, Expansion tool with increasing diameter, which mimics balloon expansion.

would not be successful. Figure 6, A, shows that the sensitivity of LCAh increases steadily from $0 \%$ at an LCAh cutoff of $7 \mathrm{~mm}$ to a $100 \%$ sensitivity at LCAh cutoff at $12 \mathrm{~mm}$. Specificity of LCAh, on the other hand, drops steadily from $100 \%$ at $7 \mathrm{~mm}$ to $0 \%$ at a cutoff of $19 \mathrm{~mm}$. The crossover point for sensitivity and specificity for LCAh as an optimal predictor of coronary obstruction was at $10 \mathrm{~mm}$ with approximately $60 \%$ sensitivity and

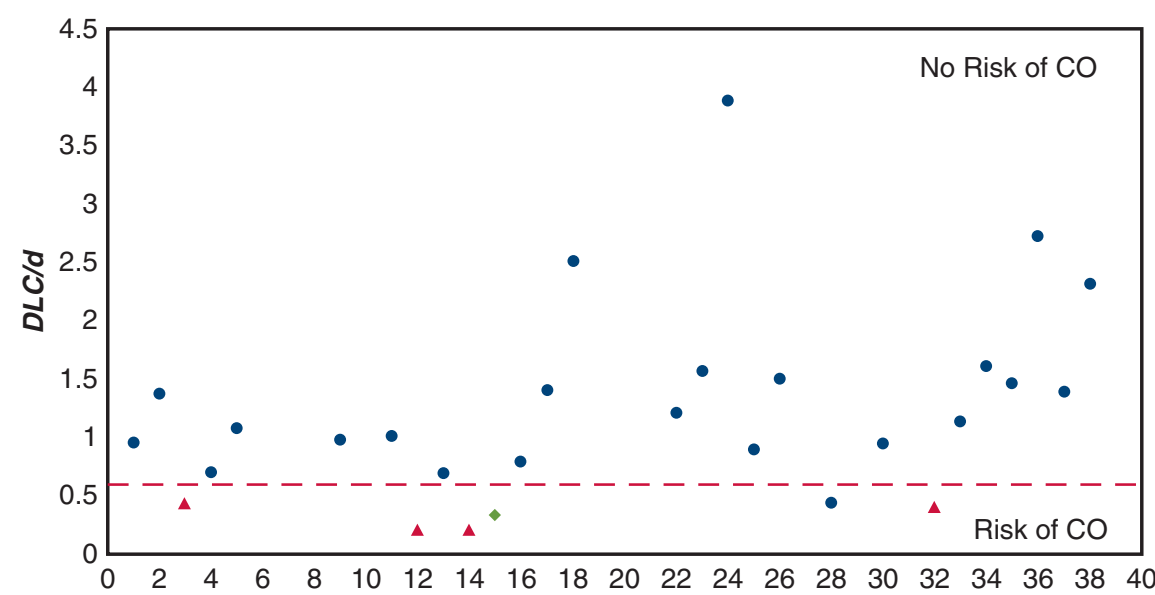

FIGURE 4. Study population characterized by 3-dimensional predictive model (DLC/d $<0.7$ ) used to predict left coronary artery obstruction before transcatheter aortic valve replacement. Blue dots represent the patients who were be approved for transcatheter aortic valve replacement under these suggested guidelines, red triangles represent those who were not approved and received other treatment, and the green diamond represents the 1 patient in whom transcatheter aortic valve replacement resulted in coronary obstruction (the model was not computed prior). DLC/d, Cusp to coronary ostium distance indexed with coronary artery diameter; $\mathrm{CO}$, Coronary obstruction. 

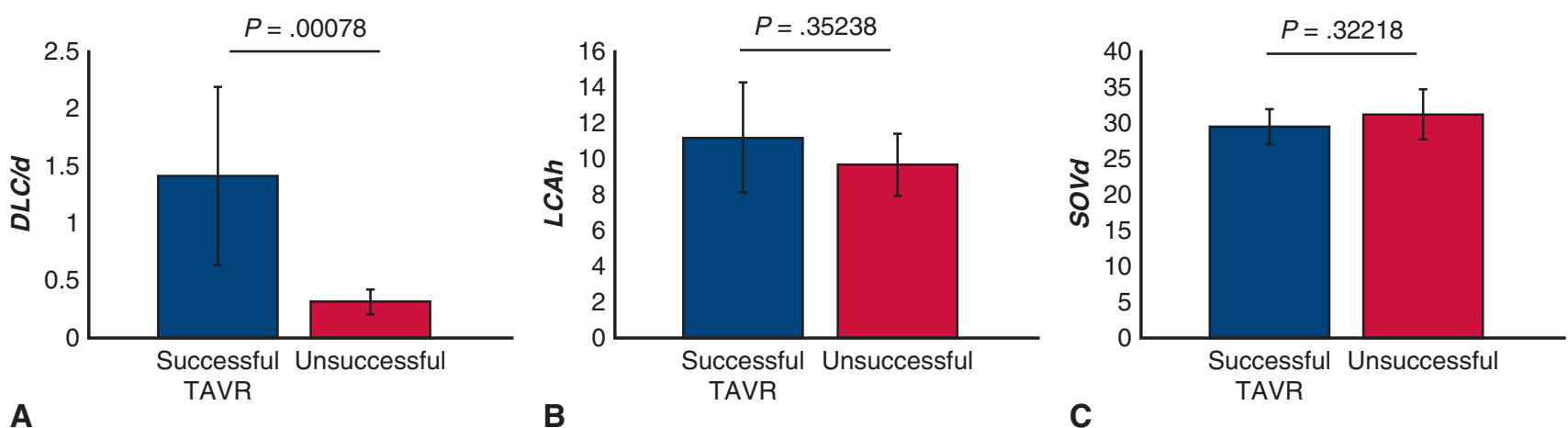

FIGURE 5. The mean and standard deviations of the parameter values (A) DLC/d, (B) coronary artery height (h), and (C) sinus of Valsalva diameter (SOVd) for those high-risk patients who successfully received a transcatheter aortic valve replacement without coronary obstruction compared with those who did not receive a transcatheter aortic valve replacement successfully. A significant difference between the 2 groups was found for the DLC/d parameter at significance level .05 . Neither h nor SOVd was significantly different between the groups. $D L C / d$, Cusp to coronary ostium distance indexed with coronary artery diameter; TAVR, transcatheter aortic valve replacement; $L C A h$, left coronary artery height; $S O V d$, sinus of Valsalva diameter.

specificity. The sensitivity and specificity of SOVd as an independent predictor of unsuccessful TAVR is shown in Figure $6, B$. The sensitivity increases from $0 \%$ at SOVd of $28 \mathrm{~mm}$ to $100 \%$ at $38 \mathrm{~mm}$. Specificity drops from $100 \%$ at $26 \mathrm{~mm}$ to $0 \%$ at $38 \mathrm{~mm}$. The optimal crossover point occurs approximately at $30.5 \mathrm{~mm}$ with a sensitivity and specificity of $40 \%$. With respect to sensitivity and specificity of the 3D computational parameter DLC/d (Figure $6, C$ ), the sensitivity is $0 \%$ at a value of 0.2 and increases to $100 \%$ at a value of 0.45 . The specificity drops from $100 \%$ at a value of 0.4 to $66 \%$ at a value of 1.0 . The optimal crossover point is slightly below 0.45 with a sensitivity and specificity of $96 \%$. There was a range of DLC/d from 0.45 to 0.70 for which the sensitivity and specificity exceed $95 \%$.

\section{DISCUSSION}

Although the prevalence of coronary obstruction during TAVR procedure is rather low $(<1 \%)$, it is a time-sensitive and life-threatening complication. To minimize cases of coronary obstruction after TAV deployment, studies have introduced safety guidelines that attempt to use geometric factors of patient aortic root geometry before implantation to assess patient risk. ${ }^{20,30-34}$ In one such study, Ribeiro and colleagues ${ }^{20}$ reported that the average coronary ostium height and mean SOVd were smaller for patients who experienced coronary obstruction during TAVR. These concepts were applied to a large population of patients who underwent TAVR $(n=6688$, 44 with coronary obstruction) and standard parameters, indicating the potential for coronary obstruction were coronary ostium height $<12 \mathrm{~mm}$ and SOVd $<30 \mathrm{~mm} .{ }^{20}$ In a smaller observational study, Yamamoto and colleagues $^{30}$ examined 666 cases of TAVR (10 with coronary obstruction), and created their own criteria for potential risk: coronary ostium height $<10 \mathrm{~mm}$, valve cusp length $>$ coronary ostium height, and shallow SOV
$(\mathrm{SOVd}=28.5 \mathrm{~mm})$ with massive calcification. Although both of these reports suggest coronary ostium height and SOVd are important, other features are likely overlooked. The effect of the calcific nodules, for example, was not considered as a main anatomic predictor of coronary obstruction. ${ }^{20}$ Tops and colleagues, ${ }^{33}$ Apfaltrer and colleagues, $^{31}$ and Binder and colleagues ${ }^{32}$ suggested noninvasive evaluation of aortic root using multislice $\mathrm{CT}$, aortoiliac $\mathrm{CT}$, multidetector $\mathrm{CT}$, and $3 \mathrm{D}$ modeling to provide more precise information on how aortic root geometry could play a role in complications such as coronary obstruction and paravalvar leak. Additional studies have focused on computational modeling using FEA to optimize preoperative planning of TAVR and evaluate its adverse outcomes in patient-specific geometries. ${ }^{22,23,35-38}$

Not only have we shown that safety guidelines that attempt to use geometric features of a patient's aortic root before implantation are not always accurate in flagging patients at risk of coronary obstruction, but also that they reduce the number of patients who can safely undergo TAVR without coronary obstruction.

Computational modeling is an emerging trend to advance the field of heart valve replacement. ${ }^{39}$ In this study, we evaluated the predictive capacity of existing methods and proposed a novel method for the investigation of coronary obstruction risk in patients with severe aortic stenosis before TAVR who were flagged as being at risk based on conventional predictive guidelines. The novel method utilizes 3D reconstructed patient geometry for simulation of TAV deployment using FEA. The current guidelines for determining high risk of coronary obstruction include SOVd $<30 \mathrm{~mm}^{20}$ and coronary ostium height $>12 \mathrm{~mm},{ }^{40,41}$ although these guidelines are not consistently recognized throughout US hospitals. Individual transcatheter valve manufacturers impose their own guidelines. For example, the manufacturers of 

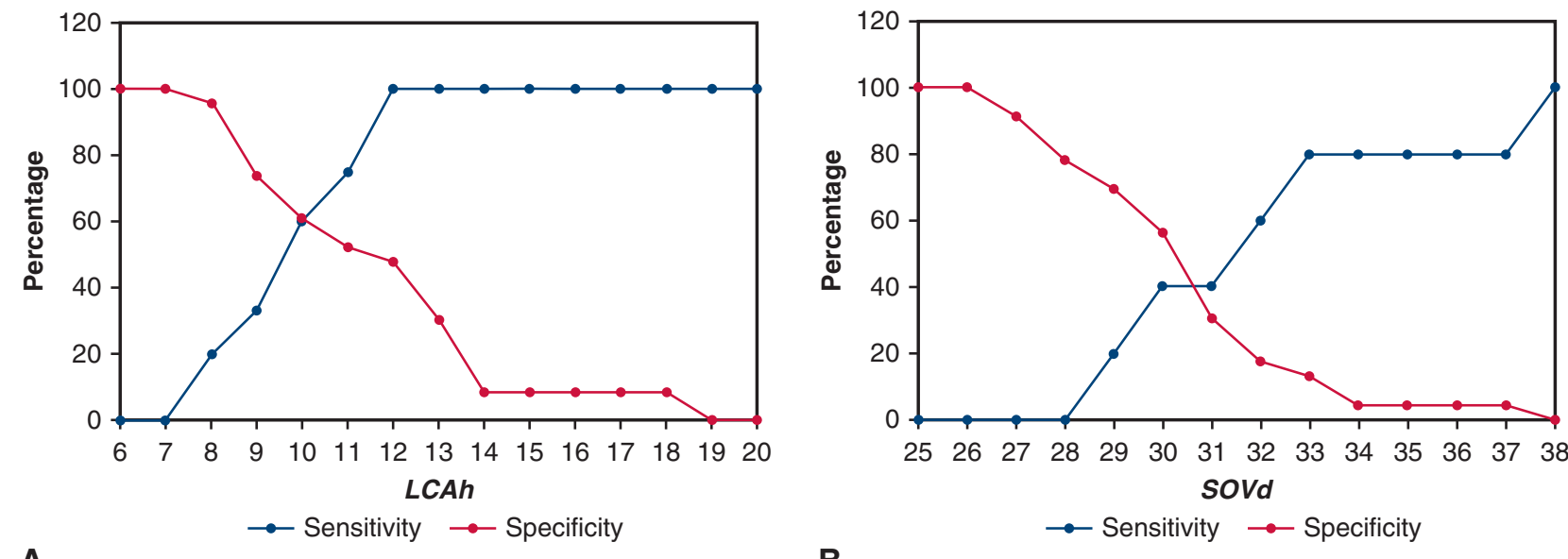

A

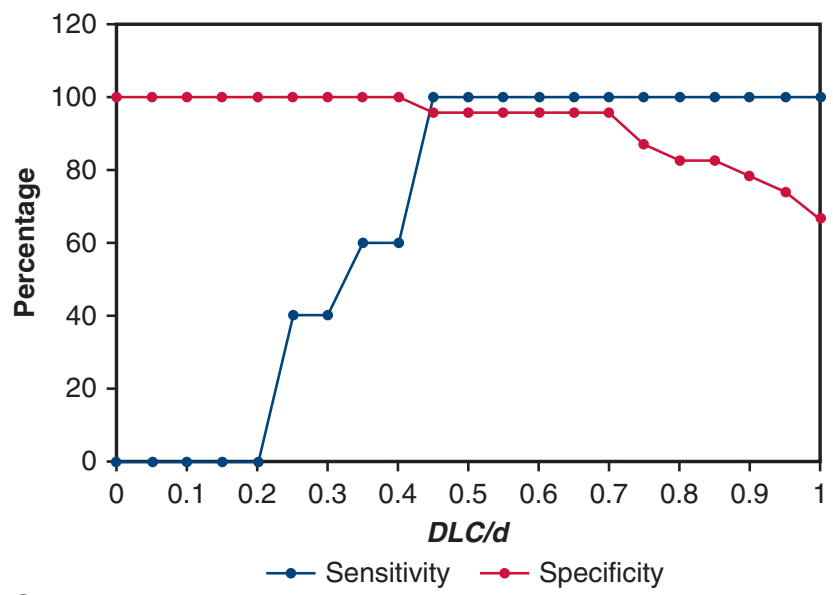

C

FIGURE 6. A-C, Sensitivity and specificity curves generated for each of the three parameters, DLC/d, coronary artery height (h), and sinus of Valsalva diameter $(S O V d)$, to predict whether transcatheter aortic valve replacement within this high-risk patient population would result in coronary obstruction. $L C A h$, Left coronary artery height; SOVd, sinus of Valsalva diameter; $D L C / d$, cusp to coronary ostium distance indexed with coronary artery diameter.

CoreValve Evolut R \& PRO (Medtronic, Dublin, Ireland) suggest that $\mathrm{SOVd} \geq 27 \mathrm{~mm}$ and $\geq 29 \mathrm{~mm}$ should be included (for the 26-mm and 29-mm Evolut R \& PRO, respectively). Similar to the SOVd guideline, coronary ostium height $\geq 14 \mathrm{~mm}$ is recommended by the CoreValve Evolut R \& PRO manufacturers. The latter guideline would exclude all but 2 of the patients in our study population, many of whom who safely underwent TAVR without coronary obstruction. On the other hand, the patientspecific 3D predictive model captures a much more accurate representation of the TAVR procedure and captures the final configuration of the TAV stent along with native cusp and aortic wall precisely. Based on our findings, the parameter DLC/d $>0.7$ when coronary ostium height $<14 \mathrm{~mm}$ and/or SOVd $<30 \mathrm{~mm}$ should be considered as patients who are not actually at high risk for left coronary obstruction, whereas patients who have DLC/d $<0.5$ are at severe risk of coronary obstruction and TAVR should not be attempted. Further studies are needed to resolve the patients where DLC/d lies between 0.5 and 0.7 . Until then, these patients should be approached with caution with potential coronary protection strategies. Further, for patients with coronary ostium height $>14 \mathrm{~mm}$ and SOVd $>30 \mathrm{~mm}$, there does not seem to be any benefit to performing simulations to evaluate DLC/d because there was no known case of coronary obstruction in this group. Furthermore, the objective of the new computational model was not to replace the current guidelines but to augment the predictive power.

\section{Limitations}

We are not looking at coronary obstruction from conduit of TAV, which can occur due to malpositioning (supra-annular) of the TAV. Additionally, right coronary obstruction was not evaluated, because obstruction of the right coronary artery is much less prevalent compared with LCA ${ }^{8-10}$ However, the 3D model is likely applicable to right coronary artery for preoperative risk assessment 
of coronary obstruction. Another limitation of the study is the small number of patients evaluated for coronary obstruction, which is due to its relatively rare occurrence.

\section{CONCLUSIONS}

We have successfully developed a highly accurate model to screen patients for possible coronary obstruction during TAVR based on criteria that can be readily calculated from current pre-TAVR CT angiographic imaging utilizing 3D FEA analysis. Neither coronary ostium height nor SOVd is predictive of coronary obstruction when considering high-risk patients with coronary ostium height $<14 \mathrm{~mm}$ and/or SOVd $<30 \mathrm{~mm}$. However, the new parameterDLC/d-is predictive of coronary obstruction for the same high-risk group. The performance of DLC/d was validated in vitro and clinically. Results indicate that a significantly high fraction of patients with coronary ostium height $<14 \mathrm{~mm}$ and/or SOVd $<30 \mathrm{~mm}$ can be safely treated with TAVR if assessed with DLC/d compared with the current guidelines using SOVd and coronary ostium height alone. These findings shed light on a rare but significant potential complication during TAVR and can assist heart teams in the decision-making process before TAVR procedures.

\section{Conflict of Interest Statement}

Dr Crestanello has received grants from Medtronic, Boston Scientific, and Abbot in addition to being part of the advisory board of Medtronic. Dr Dasi and Ms Heitkemper report having 2 patents filed on novel polymeric heart valves. Dr Dasi and Mr Azimian have a patent filed on 3D computational modeling for TAVR. All other authors have nothing to disclose with regard to commercial support.

\section{References}

1. Leon MB, Smith CR, Mack MJ, Makkar RR, Svensson LG, Kodali SK, et al. Transcatheter or surgical aortic-valve replacement in intermediate-risk patients. N Engl J Med. 2016;374:1609-20.

2. Masson J-B, Kovac J, Schuler G, Ye J, Cheung A, Kapadia S, et al. Transcatheter aortic valve implantation: review of the nature, management, and avoidance of procedural complications. JACC Cardiovasc Interv. 2009;2:811-20.

3. Smith CR, Leon MB, Mack MJ, Miller DC, Moses, Svensson LG, et al. Transcatheter versus surgical aortic-valve replacement in high-risk patients. N Engl J Med. 2011;364:2187-98.

4. Dasi LP, Hatoum H, Kheradvar A, Zareian R, Alavi SH, Sun W, et al. On the mechanics of transcatheter aortic valve replacement. Ann Biomed Eng. 2017; 45:310-31.

5. Rodés-Cabau J. Transcatheter aortic valve implantation: current and future approaches. Nat Rev Cardiol. 2012;9:15.

6. Dvir D, Leipsic J, Blanke P, Ribeiro HB, Kornowski R, Pichard A, et al. Coronary obstruction in transcatheter aortic valve-in-valve implantation: preprocedural evaluation, device selection, protection, and treatment. Circ Cardiovasc Interv. $2015 ; 8$.

7. Gurvitch R, Tay EL, Wijesinghe N, Ye J, Nietlispach F, Wood DA, et al. Transcatheter aortic valve implantation: lessons from the learning curve of the first 270 high-risk patients. Catheter Cardiovasc Interv. 2011;78:977-84.

8. Mizote I, Conradi L, Schäfer U. A case of anomalous left coronary artery obstruction caused by lotus valve implantation. Catheter Cardiovasc Interv. 2017;90:1227-31.
9. Gökdeniz T, Aykan AÇ, Ağaç MT, Dağdelen S, Çelik Ş. Concomitant complete atrioventricular block and left main coronary artery occlusion during transcatheter aortic valve implantation. Heart Lung Circ. 2013;22:1048-50.

10. Dağdelen S, Karabulut H, Alhan C. Acute left main coronary artery occlusion following TAVI and emergency solution. Anadolu Kardiyol Derg. 2011;11:747.

11. Zierer A, Wimmer-Greinecker G, Martens S, Moritz A, Doss M. The transapical approach for aortic valve implantation. J Thorac Cardiovasc Surg. 2008;136: 948-53.

12. Flecher EM, Curry JW, Joudinaud TM, Kegel CL, Weber PA, Duran CM Coronary flow obstruction in percutaneous aortic valve replacement. An in vitro study. Eur J Cardiothorac Surg. 2007;32:291-5.

13. Gurvitch R, Cheung A, Bedogni F, Webb JG. Coronary obstruction following transcatheter aortic valve-in-valve implantation for failed surgical bioprostheses. Catheter Cardiovasc Interv. 2011;77:439-44.

14. Bagur R, Dumont E, Doyle D, Larose E, Lemieux J, Bergeron S, et al. Coronary ostia stenosis after transcatheter aortic valve implantation. JACC Cardiovasc Interv. 2010;3:253-5.

15. Crimi G, Passerone G, Rubartelli P. Transapical aortic valve implantation complicated by left main occlusion. Catheter Cardiovasc Interv. 2011;78:656-9.

16. Maddox TM, Stanislawski MA, Grunwald GK, Bradley SM, Ho PM, Tsai TT, et al. Nonobstructive coronary artery disease and risk of myocardial infarction. JAMA. 2014;312:1754-63.

17. Levine GN, Bates ER, Blankenship JC, Bailey SR, Bittl JA, Cercek B, et al. 2011 ACCF/AHA/SCAI guideline for percutaneous coronary intervention: a report of the American College of Cardiology Foundation/American Heart Association task force on practice guidelines and the Society for Cardiovascular Angiography and Interventions. J Am Coll Cardiol. 2011;58:e44-122.

18. Moore BL, Dasi LP. Coronary flow impacts aortic leaflet mechanics and aortic sinus hemodynamics. Ann Biomed Eng. 2015;43:2231-41.

19. Hatoum H, Dollery J, Lilly SM, Crestanello JA, Dasi LP. Implantation depth and rotational orientation effect on valve-in-valve hemodynamics and sinus flow. Ann Thorac Surg. 2018;106:70-8.

20. Ribeiro HB, Webb JG, Makkar RR, Cohen MG, Kapadia SR, Kodali S, et al. Predictive factors, management, and clinical outcomes of coronary obstruction following transcatheter aortic valve implantation: insights from a large multicenter registry. J Am Coll Cardiol. 2013;62:1552-62.

21. Hatoum H, Lilly SM, Crestanello J, Dasi LP. A case study on implantation strategies to mitigate coronary obstruction in a patient receiving transcatheter aortic valve replacement. J Biomech. 2019;89:115-8.

22. Bosmans B, Famaey N, Verhoelst E, Bosmans J, Vander Sloten J. A validated methodology for patient specific computational modeling of self-expandable transcatheter aortic valve implantation. J Biomech. 2016;49:2824-30.

23. Auricchio F, Conti M, Morganti S, Reali A. Simulation of transcatheter aortic valve implantation: a patient-specific finite element approach. Comput Methods Biomech Biomed Engin. 2014;17:1347-57.

24. Billiar KL, Sacks MS. Biaxial mechanical properties of the natural and glutaraldehyde treated aortic valve cusp - part I: experimental results. J Biomech Eng. 2000;122:23-30.

25. Hatoum H, Dollery J, Lilly SM, Crestanello J, Dasi LP. Impact of patient-specific morphologies on sinus flow stasis in transcatheter aortic valve replacement: an in-vitro study. J Thorac Cardiovasc Surg. 2019;157:540-9.

26. Hatoum H, Yousefi A, Lilly S, Maureira P, Crestanello J, Dasi LP. An in vitro evaluation of turbulence after transcatheter aortic valve implantation. J Thorac Cardiovasc Surg. 2018;156:1837-48.

27. Lalkhen AG, McCluskey A. Clinical tests: sensitivity and specificity. Contin Educ Anaesth Crit Care Pain. 2008;8:221-3.

28. Hatoum H, Moore BL, Dasi LP. On the significance of systolic flow waveform on aortic valve energy loss. Ann Biomed Eng. 2018;46:2102-11.

29. Hatoum H, Heim F, Dasi LP. Stented valve dynamic behavior induced by polyester fiber leaflet material in transcatheter aortic valve devices. J Mech Behav Biomed Mater. 2018;86:232-9.

30. Yamamoto M, Shimura T, Kano S, Kagase A, Kodama A, Koyama Y, et al Impact of preparatory coronary protection in patients at high anatomical risk of acute coronary obstruction during transcatheter aortic valve implantation. Int J Cardiol. 2016;217:58-63.

31. Apfaltrer P, Schymik G, Reimer P, Schroefel H, Sueselbeck T, Henzier T, et al Aortoiliac CT angiography for planning transcutaneous aortic valve implantation: aortic root anatomy and frequency of clinically significant incidental findings. Am J Roentgenol. 2012;198:939-45.

32. Binder RK, Webb JG, Willson AB, Urena M, Hansson NC, Norgaard BL, et al. The impact of integration of a multidetector computed tomography 
annulus area sizing algorithm on outcomes of transcatheter aortic valve replacement: a prospective, multicenter, controlled trial. J Am Coll Cardiol. 2013;62:431-8.

33. Tops LF, Wood DA, Delgado V, Schuijf JD, Mayo JR, Pasupati S, et al Noninvasive evaluation of the aortic root with multislice computed tomography: implications for transcatheter aortic valve replacement. JACC Cardiovasc Imaging. 2008;1:321-30.

34. Akhtar M, Tuzcu EM, Kapadia SR, Svensson LG, Greenberg RK, Roselli EE, et al. Aortic root morphology in patients undergoing percutaneous aortic valve replacement: evidence of aortic root remodeling. J Thorac Cardiovasc Surg. 2009;137:950-6

35. Capelli C, Bosi G, Cerri E, Nordmeyer J, Odenwald T, Bonhoeffer P, et al Patient-specific simulations of transcatheter aortic valve stent implantation. Med Biol Eng Comput. 2012;50:183-92.

36. Capelli C, Taylor AM, Migliavacca F, Bonhoeffer P, Schievano S. Patient-specific reconstructed anatomies and computer simulations are fundamental for selecting medical device treatment: application to a new percutaneous pulmonary valve. Philos Trans A Math Phys Eng Sci. 2010;368: 3027-38.
37. Morganti S, Conti M, Aiello M, Valentini A, Mazzola A, Reali A, et al Simulation of transcatheter aortic valve implantation through patient-specific finite element analysis: two clinical cases. J Biomech. 2014;47:2547-55.

38. Wang Q, Sirois E, Sun W. Patient-specific modeling of biomechanical interaction in transcatheter aortic valve deployment. J Biomech. 2012;45:1965-71.

39. Kheradvar A, Groves EM, Falahatpisheh A, Mofrad MK. Hamed Alavi S, Tranquillo R, et al. Emerging trends in heart valve engineering: part iv. Computational modeling and experimental studies. Ann Biomed Eng. 2015;43:2314-33.

40. Achenbach S, Delgado V, Hausleiter J, Schoenhagen P, Min JK, Leipsic JA. SCCT expert consensus document on computed tomography imaging before transcatheter aortic valve implantation (TAVI)/transcatheter aortic valve replacement (TAVR). J Cardiovasc Comput Tomogr. 2012;6:366-80.

41. Holmes DR Jr, Mack MJ, Kaul S, Agnihotri A, Alexander KP, Bailey SR, et al. 2012 ACCF/AATS/SCAI/STS expert consensus document on transcatheter aortic valve replacement. J Am Coll Cardiol. 2012;59:1200-54.

Key Words: TAVR, coronary obstruction, calcification, FEA, CFD, patient-specific 

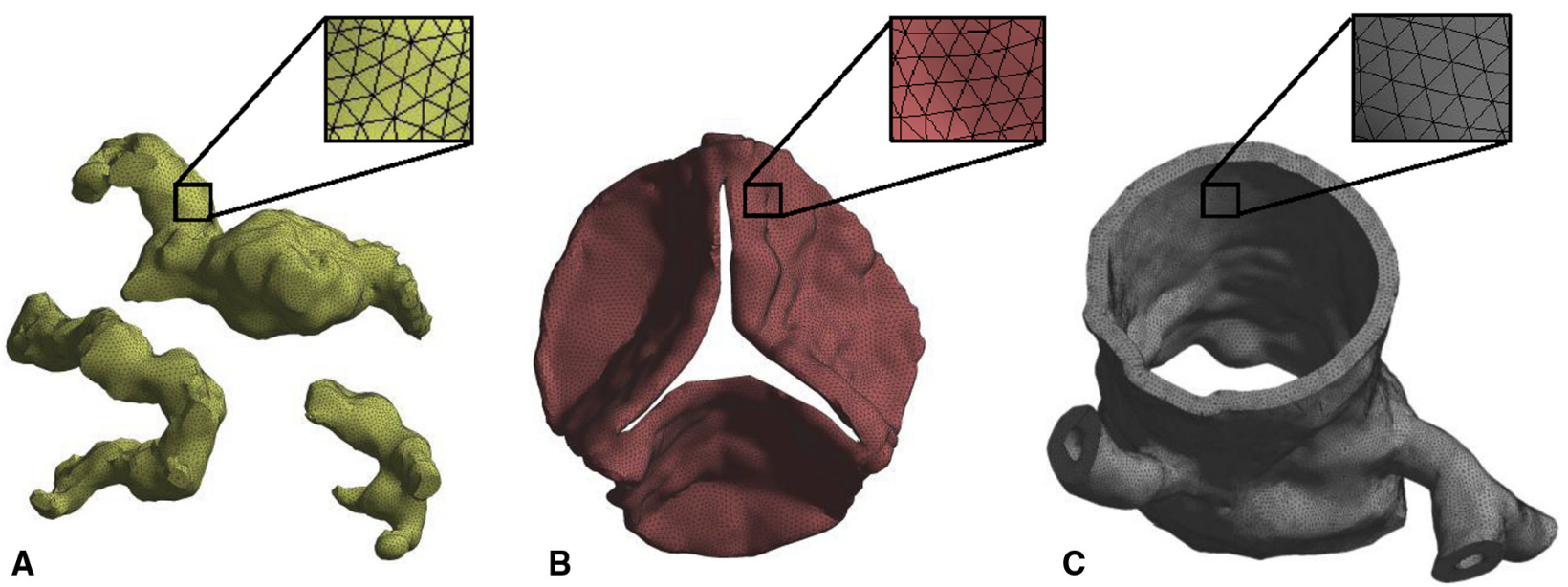

FIGURE E1. All the patient-specific tissues, including aortic wall, leaflets, and calcium nodules were discretized using an explicit 4-node linear tetrahedron element, whereas the cylindrical stent was discretized using hexahedron elements. The total number of mesh elements for each patient was $35,580,2835$, and 3240 elements for the wall, the leaflets, and the calcium nodule, respectively. A, Calcification on the leaflets. B, The 3 cusps. C, Aortic root, including coronary arteries. 


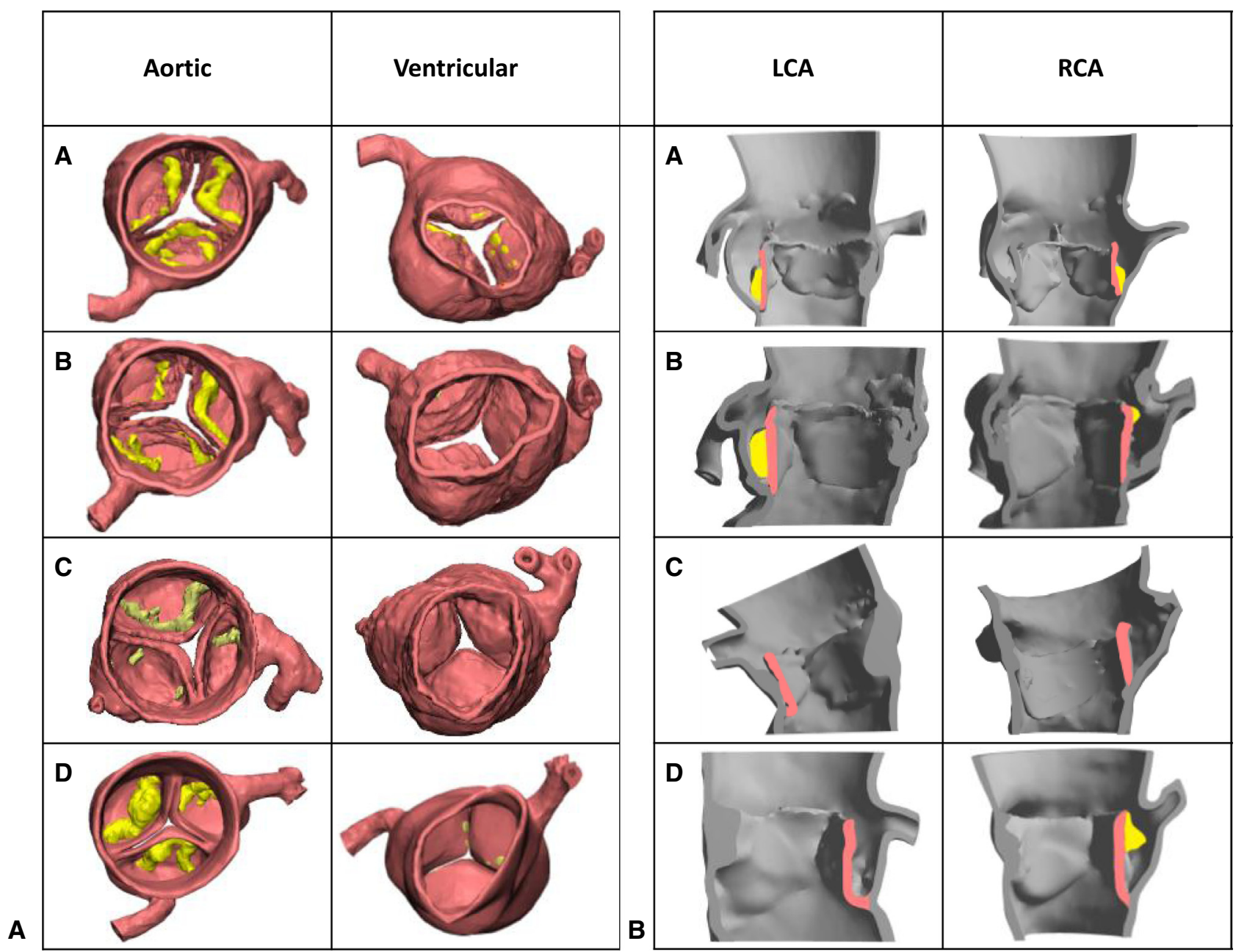

FIGURE E2. A, Aortic and ventricular views of the reconstructed patient aortic roots. The aortic views are oriented with the commissure of non- and left coronary cusps at the top. In the ventricular views, the top commissure corresponds to the left and right coronary cusps. Calcific nodules (yellow) are highlighted for each patient and the morphology of the calcific lesions are noted to be highly patient specific. B, The cross-sectional views of finite element analysis generated geometries after transcatheter aortic valve replacement for the respective patients shown in A. Cross-sectional views depict both left and right coronary ostia to illustrate the final position of leaflets and calcific lesion relative to the respective coronary ostia center. $L C A$, Left coronary artery; $R C A$, right coronary artery. 


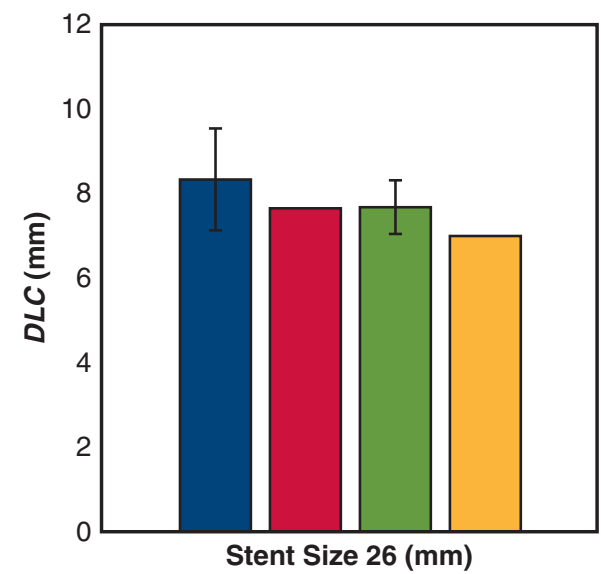

Experiment w/o calcium $\square$ Simulation w/o Calcium

$\square$ Experiment $\mathrm{w} /$ calcium $\square$ Simulation $\mathrm{w} /$ calcium

FIGURE E3. Comparison between in vitro validation technique for stent size $26 \mathrm{~mm}$ and the finite element analysis simulation with simulated transcatheter aortic valve expanded to a diameter of $26 \mathrm{~mm}$. The final measurements are plotted with the $y$-axis showing the measured distance between native leaflets to the left coronary ostium $(D L C)$.

TABLE E1. List of material properties for aortic root geometry, including aortic wall, leaflets, and calcium nodules

\begin{tabular}{lccccc}
\hline Aortic root geometry & $\boldsymbol{\mu}(\mathbf{P K a})$ & $\lambda(\mathbf{P K a})$ & $\boldsymbol{\rho}\left(\mathbf{k g} / \mathbf{m}^{\mathbf{3}}\right)$ & $\boldsymbol{E}(\mathbf{M p a})$ & - \\
\hline Wall & 500 & 10,000 & 1000 & - & - \\
Leaflets & 1000 & 10,000 & 1000 & 1200 & 80 \\
Calcium nodules & - & - & 0.3 \\
\hline
\end{tabular}

